\title{
A Distributed and Collective Approach for Curved Object-Based Range Image Segmentation
}

\author{
Smaine Mazouzi ${ }^{1}$, Zahia Guessoum ${ }^{2}$, and Fabien Michel $^{3}$ \\ 1 Dép. d'informatique, Université de Skikda, BP 26, Route ElHadaik, 21000, Algérie \\ mazouzi_smaine@yahoo.fr \\ 2 LIP6, Université de Paris 6, 104, av. du Président Kennedy, 75016, Paris, France \\ zahia.guessoum@lip6.fr \\ 3 LIRMM 161 rue Ada 34392 Montpellier Cedex 5, France \\ fmichel@lirmm.fr
}

\begin{abstract}
In this paper, we use multi-agent paradigm in order to propose a new method of image segmentation. The images considered in this work are the range images which can contain at once polyhedral and curved objects. The proposed method uses a multi-agent approach where agents align the region borders to the surrounding surfaces which make emerging a collective segmentation of the image. The agents move on the image and when they arrive on the pixels of a region border they align these pixels to their respective surfaces. The resulting competitive alignment allows at once the emergence of the image edges and the disappearance of the noise regions. The test results obtained with real images show a good potential of the new method for accurate image segmentation.
\end{abstract}

Keywords: Image segmentation, Multi-agent systems, Curved Object, Range image.

\section{Introduction}

A range image represents the visible surface of a three-dimensional scene, where at each pixel of the image is stored the depth of the corresponding point of the scene. Range images are mainly used in recognition of $3 \mathrm{D}$ objects in robotic vision, because the $3 \mathrm{D}$ information, which is required for object recognition is immediately available. However, these images are recognized as being highly noised images [3] and are consequently hard to segment and to interpret. Several authors proposed methods of segmentation for this class of images [48]. However, most of these authors limited themselves to the images based on polyhedral objects. For these last ones, the detection of the regions of interest is widely easier compared with images containing curved objects [4.

Aiming at segmenting range images with both polyhedral and curved objects, we have appealed to multi-agent paradigm and more particularly to the reactive agents [2]. We have used simple agents with weak granularity and having simple

E. Bayro-Corrochano and J.-O. Eklundh (Eds.): CIARP 2009, LNCS 5856, pp. 201 208, 2009.

(C) Springer-Verlag Berlin Heidelberg 2009 
behavior which based on the mechanism of stimulus-reaction. The agents move on the image and act on the met pixels. An agent aligns the first not homogeneous pixel to the surface on which it moves. So, pixels belonging to the noise regions or situated on the circumferences of surfaces are systematically aligned to the surfaces which surround them. We show that the alternative alignment of pixels on the borders of regions allows preserving edges. On the other hand, because there is no pixel alignment within the noise regions, theses regions progressively disappear. This collective action of the agents allows the emergence of edges and the disappearance of the noise regions.

Most of the methods having used the agent paradigm 1179106 are supervised and consequently they can be applied only to the images for which they were conceived. The method proposed in this paper is unsupervised. It makes no assumption on the number and the shape of the surfaces which compose objects in the image. This allows the adaptation of the method to any type of images by defining the criteria of homogeneity of the regions composing the treated images. The distribution of treatments and decision which characterize our approach, as well as the weak coupling of the reactive agents, offers a parallel method well suitable for real-time image interpretation. The experimentation of the method by using real images allowed to validate the new approach and to show its potential for an accurate and effective segmentation of range images.

The reminder of the paper is organized as follows: in Section 2 we present the main approaches having used multi-agent systems for image segmentation. Section 3 is devoted to the proposed approach. We present at first the criterion of homogeneity curved surfaces adopted in our case. Then, we introduce the principle of the multi-agent system and we show how a collective segmentation emerges form the simple and reactive behaviors of the agents. Our test results are introduced in the section 5 , in which we show the the parameter selection and we comment on the obtained results. Finally, a conclusion summarizes our work and underlines its potential extensions.

\section{Multi-agent Approches for Image Segmentation}

Since the publication of the first works proposing multi-agent approaches for image processing, the cooperation and the interaction between agents represented the new contributions to deal with the problem of ambiguity which characterizes image visual data [17].

In the category of edge based segmentation methods using a reactive approach, Ballet et al. 1] have defined a multi-agent system for edge detection and following in 2D images. In this system an agent is situated on an extremum of the luminance gradient, then follows the crest of the edge and records its path in a shared memory. The previously recorded edge segments are used by other agents to initialize new edge segments or finish others. The method proposed by the authors is based on an original approach of parallel and distributed edge detection. However, the authors have not considered any mechanism of cooperation or coordination to strengthen the detection and improve the segmentation results. 
The same authors [10] have resumed later the same approach and have extended it by using the multi-agent language oRis. By considering the a priori knowledge on the topology of regions in certain types of images, the agents follow the lines of gradient extremum. By considering two types of image regions, two categories of agents named respectively darkening agents and lightning agents are defined. According to their category, the agents follow respectively the dark regions or the light regions. By remaining on crest lines, the agents strengthen the difference of contrast between the pairs of neighboring regions. The proposed system is well dedicated to images containing roof edges (detected by the discontinuity of the first derivative of the image). Indeed, theses edges characterize the considered images by the authors.

The previous multi-agent approaches like most of edge based approches, have proceeded to detection without any region representation. This does not facilitate the region based segmentation of the treated images.

In the category of region based methods, Liu et al. 7] have used a reactive agent based system for the segmentation of Magnetic Resonance Images (MRI) of the brain. The authors used four types of agents, which correspond to the four tissues in the pathological cerebral matter. A first generation of agents is initialized inside the various tissues. Then, when every agent recognizes its region, it creates offspring agents and places them so that they are lucky to find more other homogeneous pixels. For the same type of images, Richard and his co-authors 9] proposed a multi-agent system were agents are organized hierarchically as follows: 1) One global control agent which partition the image volume in partial volumes; 2) Local control agents, allocated each one to a partial volume; 3) tissue dedicated agents, which work under the control of a local control agent, to segment the tissue inside a partial volume. So, the detection is performed at the lowest level by the tissue dedicated agents, then synthetized by the local control agents and the global control agent.

Like most of the region based approaches of image segmentation, the previous two works follow supervised approaches, where it is necessary to know the number of regions and their shapes. We introduce into this paper a new approach which is general and unsupervised for range image segmentation. We show that the competitive alignment of edges performed by reactive agents allows the emergence of a collective segmentation of an image. The proposed approach is unsupervised. It makes no preliminary assumption on the number or the nature of the regions which form the images to be segmented.

\section{Image Segmentation by Edge Emergence}

In order to do not make any assumption on the shape of regions, we have used the directional curvatures of surface, as the homogeneity criterion. Let a pixel $(x, y)$, the two directional curvatures, respectively horizonal $C_{h}$ and vertical $C_{v}$, are defined as follows:

$$
C_{h}(x, Y)=-\frac{I^{\prime \prime}(x, .)}{1+I^{\prime 2}(x, .)}
$$




$$
C_{v}(X, y)=-\frac{I^{\prime \prime}(., y)}{1+I^{\prime 2}(., y)}
$$

where $I^{\prime}(x,$.$) and I^{\prime \prime}(x,$.$) are the first and the second derivative of the image$ following the $x$ axis, by fixing the value of $y$ to $Y$. The same, $I^{\prime}(., y)$ and $I^{\prime \prime}(., y)$ are the first and the second derivative of the image following the $y$ axis, by fixing the value of $x$ to $X . C_{h}$ expresses the curvature projection of the surface of the plane $y=Y$, while $C_{v}$ expresses the curvature projection of the surface on the plane $x=X$. A contiguous set $R$ of pixels $\left\{\left(x_{k}, y_{k}\right), k \in R\right\}$ is considered homogenous, and represents an image region, if all of its pixels have, according to a given threshold $T r_{c}$, the same directional curvatures:

$$
\forall k, l \in R ;\left|C_{h}\left(x_{k}, y_{k}\right)-C_{h}\left(x_{l}, y_{l}\right)\right|<\operatorname{Tr}_{c} \wedge\left|C_{v}\left(x_{k}, y_{k}\right)-C_{v}\left(x_{l}, y_{l}\right)\right|<\operatorname{Tr}_{c}
$$

$\operatorname{Tr}_{c}$ is a curvature comparison threshold where the value is automatically set at the stage of parameter learning (see Section 4). So, the pixels on interest, which are situated at the borders of the true regions or are in the noise regions, are detected when one of the two curvature $C_{h}$ ou $C_{v}$ changes.

\subsection{System Dynamic}

A high number of reactive agents $(N=3500$ see Section 4) are initialized at random positions in the image. After, agents start to move within the image following random directions. An agent searchs for an homogenous region around its current position $\left(x_{c}, y_{c}\right)$, by checking the last $L$ visited pixels. The path length in pixels $L$ (set at Section 4) allows the agent to be sure that it is within a homogenous region. If these last $L$ pixels have all the same directional curvatures, the agent considers that it is within a homogenous region (CurrentRegion). It acquires then the ability to alter the image (Alteration Ability $\leftarrow$ TRUE). This ability allows the agent to modify the depths of the encountered pixels. In its future moves, it smoothes the encountered pixels if these latter belong to the surface on which it currently moves. When the agent arrives on the first non homogenous pixel, it aligns this pixel to the current surface. The agent loses its alteration ability and restarts to search for a new homogenous region. The next algorithm introduces the method "step()" executed by an agent at every step on its path.

Initialisation :

$$
\begin{aligned}
& \text { Alteration Ability } \leftarrow \text { False } \\
& \left(x_{c}, y_{c}\right) \leftarrow(\text { random }(\text { widthImg }-1), \text { random }(\text { heightImg }-1)) \\
& l \leftarrow 0 / / \text { Set the path length }
\end{aligned}
$$

\subsection{Noise Region Erasing and Edge Emergence}

A noise region in a range image is either a homogenous region with weak size, or a non homogenous region formed by noise pixels having random or aberrant 


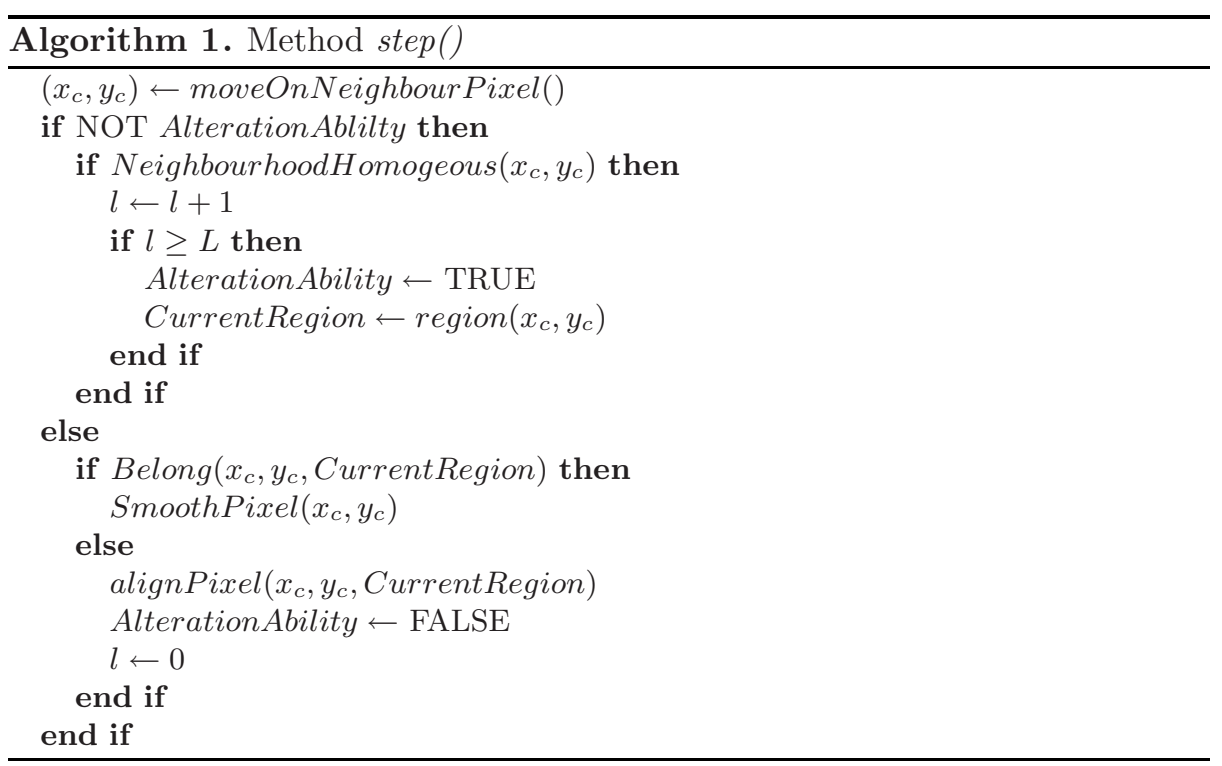

depths. In the case of a weak size, when it is crossed by an agent, this latter can not cover $L$ homogenous contiguous pixels. When the region is formed only by noise pixels, an agent when crossing it does not find at least $L$ homogenous pixels to be able to initialize a homogenous region whichever the diameter of the region. These regions are progressively erased by aligning their border pixels to the surrounding true regions. Indeed, when an agent comes on a pixel on the border of a noise region, it aligns this pixel and goes in this region. Since it could not cross $L$ contiguous homogenous pixels, it remains incapable to alter pixels within the noise region. When it leaves this region, it does not align the first encountred pixel in the homogenous surrounding region. So, the border of the noise regions is then continually aligned from outside to the surrounding true regions. The noise regions will disappear after several times agents cross theses regions. Note that one noise region is surrounded by agents of a same group. Agents of this group are those moving on the true region which surrounds the noise region.

On the other hand, a segment of a thick edge, situated between two homogenous regions, is surrounded by two groups of agents. Agents of each group are situated entirely within only one region. Agents which cross the segment align the pixels of the segment border to the region from where they come. The edge segment between the two homogenous regions is thus continually thinned by the two groups of agents (Fig. 17a and 1k).

When the edge becomes thin (one pixel wide), the agents of the two groups will become in competition to align the pixels of the thinned edge. Indeed, the pixels aligned by the agents of a giver group, let A in Fig $1 \mathrm{~b}$ are immediately realigned 


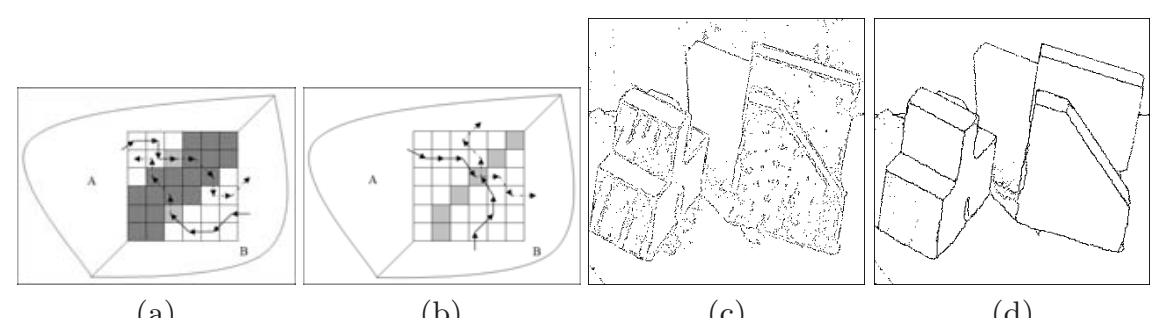

(a)

(b)

(c)

(d)

Fig. 1. Edge thinning (image abw.test.6). (a),(c) Edge pixels at $t=800$; (b),(d) Edge pixels at $t=8000$.

to the second region (B) by the agents of the second group. So, the pixels of the edge are continually switched between the two regions. Consequently, whichever the number of alignments of these pixels, they remain emergent in the image (Fig. [1). Note that this result is not coded in any agent, but it emerges from the collective action of all the agents in the image.

At the end of the process, noise regions are erased, and edges are thinned. So, a simple region growing controlled by the detected edges allows to produce a region based segmentation of the image.

\section{Experimentation}

We have used the framework proposed by Hoover et al. 3 to evaluate our approach, with range images from the set K2T containing both curved and polyhedral objects. All the images have a size of $640 \times 480$ pixels. A set of 10 range images was used to select the values of the used parameters: the number of agents $N=3500$; the path length $L=7$; and the curvature threshold $T r_{c}$. The selected value correspond to the best segmentation, expressed as the number of the regions correctly detected, according to a ground truth (GT) segmentation [3].

In order to show how the noise regions are progressively erased and how edges are thinned, we show aligned pixels at regular intervals of time $t$. Figure $2 a$ shows a sample of a range image. Figures $2 \mathrm{~b}, 2 \mathrm{k}, 2 \mathrm{~d}, 2 \mathrm{r}$ and $2 \mathrm{k}$ show the aligned pixels respectively at $t=500,3500,7500,10500$ and 13500 . As the time progresses edges are detected and thinned, and noise regions are erased.

Fig. [3] shows the average number of instances of correct detection corresponding respectively to our method (DCIS for Distributed and Collective Image Segmentation) and the method of EG (Edge based Segmentation), where authors have proposed a segmentation method for curved objects 415. Theses results are obtained with the set K2T of images containing curved objets, and are computed according to a compare tool tolerance $T$, which is used to express the comparison tolerance. We can observe that our method records scores better than those of EG for $T$ in $\{50 \%, 60 \%, 70 \%$ and $80 \%\}$. The two methods record equivalent scores for $T$ in $\{90 \%, 95 \%\}$. 


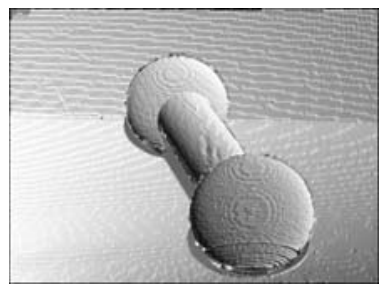

(a)

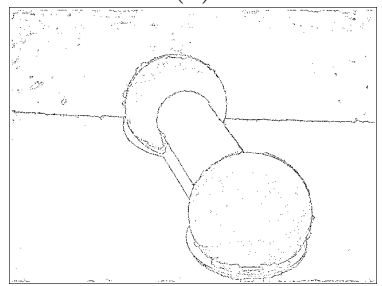

(d)

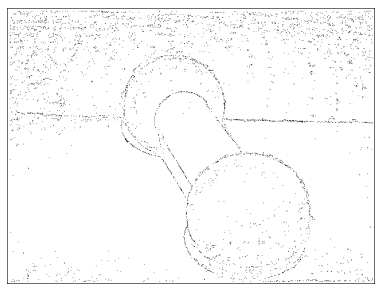

(b)

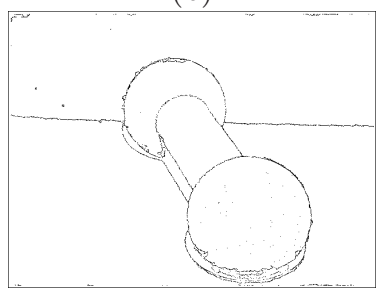

(e)

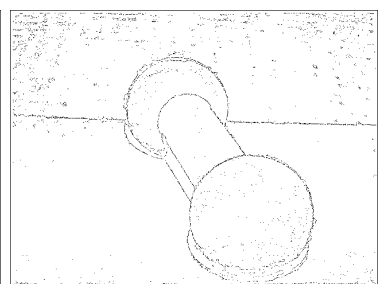

(c)

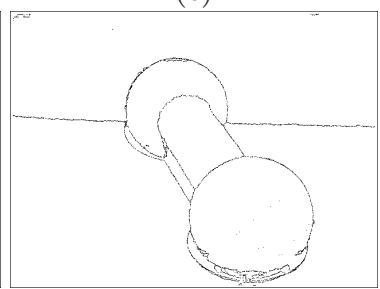

(f)

Fig. 2. Segmentation Progression. (a) Rendered Range Image; (b) at $t=500$, (c) at $t=3500$; (d) at $t=7500$; (e) at $t=10500$; (f) at $t=13500$.

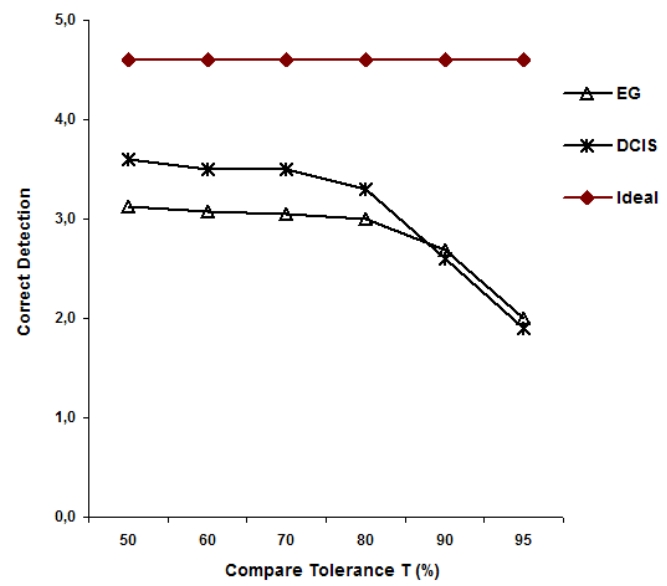

Fig. 3. Average number of correct detections in the set K2T for the two methods EG and DCIS according to $T ; 50 \% \leq T<100 \%$

\section{Conclusion}

We have proposed in this paper a new approach for edge detection and noise erasing in range images. This has allowed the segmentation of an image in its several homogenous regions. Combining the surface curvature and the competitive alignment of pixels has allowed to produce an accurate segmentation of images containing curved objects. These latter are recognized as difficult to segment in 
the case of range images. According to our approach, the detection of an edge segment results from the alternative alignment of its pixels. This alignment is performed by agents coming from the two regions. On the other hand, noise regions are progressively erased by the continuous alignment of the pixels of their borders to the homogenous surrounding regions. We think that the proposed approach could be applied to other types of images. For this, it's necessary to define a homogeneity criterion of regions in treated images.

\section{References}

1. Ballet, P., Rodin, V., Tisseau, J.: Edge detection using a multiagent system. In: 10th Scandinavian Conference on Image Analysis, Lapeenranta, Finland, pp. 621$626(1997)$

2. Ferber, J.: Les systèmes multi-agents : vers une intelligence collective. Informatique, Intelligence Artificielle. Inter Éditions (1995)

3. Hoover, A., Jean-Baptiste, G., Jiang, X., Flynn, P.J., Bunke, H., Goldgof, D.B., Bowyer, K.W., Eggert, D.W., Fitzgibbon, A.W., Fisher, R.B.: An experimental comparison of range image segmentation algorithms. IEEE Transactions on Pattern Analysis and Machine Intelligence 18(7), 673-689 (1996)

4. Jiang, X., Bunke, H.: Edge detection in range images based on Scan Line approximation. Computer Vision and Image Understanding 73(2), 183-199 (1999)

5. Jiang, X.: Recent advances in range image segmentation. In: Selected Papers from the International Workshop on Sensor Based Intelligent Robots, London, UK, pp. 272-286. Springer, Heidelberg (1999)

6. Jones, J., Saeed, M.: Image enhancement, an emergent pattern formation approach via decentralised multi-agent systems. Multiagent and Grid Systems Journal (ISO Press) Special Issue on Nature inspired systems for parallel, asynchronous and decentralised environments 3(1), 105-140 (2007)

7. Liu, J., Tang, Y.Y.: Adaptive image segmentation with distributed behavior-based agents. IEEE Transactions on Pattern Analysis and Machine Intelligence 21(6), 544-551 (1999)

8. Mazouzi, S., Batouche, M.: A new bayesian method for range image segmentation. In: Yuille, A.L., Zhu, S.-C., Cremers, D., Wang, Y. (eds.) EMMCVPR 2007. LNCS, vol. 4679, pp. 453-466. Springer, Heidelberg (2007)

9. Richard, N., Dojat, M., Garbay, C.: Automated segmentation of human brain MR images using a multi-agent approach. Artificial Intelligence in Medicine 30(2), 153 176 (2004)

10. Rodin, V., Benzinou, A., Guillaud, A., Ballet, P., Harrouet, F., Tisseau, J., Le Bihan, J.: An immune oriented multi-agent system for biological image processing. Pattern Recognition 37(4), 631-645 (2004) 